\title{
Analysis of an overlapping surgery policy change on costs in a high-volume neurosurgical department
}

\author{
Jian Guan, MD, Michael Karsy, MD, PhD, MSc, Andrea A. Brock, MD, MSCl, \\ William T. Couldwell, MD, PhD, John R. W. Kestle, MD, Randy L. Jensen, MD, PhD, \\ Andrew T. Dailey, MD, Erica F. Bisson, MD, MPH, and Richard H. Schmidt, MD, PhD \\ Department of Neurosurgery, Clinical Neurosciences Center, University of Utah, Salt Lake City, Utah
}

\begin{abstract}
OBJECTIVE Overlapping surgery remains a controversial topic in the medical community. Although numerous studies have examined the safety profile of overlapping operations, there are few data on its financial impact. The authors assessed direct hospital costs associated with neurosurgical operations during periods before and after a more stringent overlapping surgery policy was implemented.
\end{abstract}

METHODS The authors retrospectively reviewed the records of nonemergency neurosurgical operations that took place during the periods from June 1, 2014, to October 31, 2014 (pre-policy change), and from June 1, 2016, to October 31, 2016 (post-policy change), by any of the 4 senior neurosurgeons authorized to perform overlapping cases during both periods. Cost data as well as demographic, surgical, and hospitalization-related variables were obtained from an institutional tool, the Value-Driven Outcomes database.

RESULTS A total of 625 hospitalizations met inclusion criteria for cost analysis; of these, 362 occurred prior to the policy change and 263 occurred after the change. All costs were reported as a proportion of the average total hospitalization cost for the entire cohort. There was no significant difference in mean total hospital costs between the prechange and postchange period $(0.994 \pm 1.237$ vs $1.009 \pm 0.994, p=0.873)$. On multivariate linear regression analysis, neither the policy change $(p=0.582)$ nor the use of overlapping surgery $(p=0.273)$ was significantly associated with higher total hospital costs.

CONCLUSIONS A more restrictive overlapping surgery policy was not associated with a reduction in the direct costs of hospitalization for neurosurgical procedures.

https://thejns.org/doi/abs/10.3171/2018.5.JNS18569

KEYWORDS overlapping surgery; costs; neurosurgery; value-driven outcomes

$\mathrm{O}$ VERLAPPING surgery, in which one surgeon oversees two procedures, with critical portions of each operation occurring at different times but with some overlap of noncritical portions, ${ }^{3,4}$ remains a controversial topic within the medical community. When the use of overlapping surgery entered the spotlight in late $2015,{ }^{1}$ there was little peer-reviewed literature on the subject. In the years since, several studies in a variety of surgical specialties have investigated the topic, with a focus on the safety of the practice. ${ }^{7,13,18,20}$ Although patient safety is and undoubtedly should be the primary concern of the overlapping surgery debate, some observers, including members of a US Senate Finance Committee investigation, have raised concerns regarding the cost-effectiveness of the practice as well. ${ }^{21}$ Data on the costs related to overlapping procedures are scant, with only a single study published to date examining this association in spine surgeries. ${ }^{22}$

Because of the paucity of information on this important topic, we sought to investigate the influence of overlapping surgery on hospital costs across a broad array of neurosurgical procedures. Our center is uniquely situated for this investigation for two reasons. In 2015, in the face of the controversy surrounding overlapping surgery, our institution implemented more stringent guidelines regard- 
ing the scheduling of overlapping cases, which significantly reduced their incidence. Furthermore, our institution maintains an institutional tool, the Value-Driven Outcomes (VDO) tool, which was developed for value-based healthcare analysis and evaluation of costs. ${ }^{14}$ Using the VDO, we analyzed total hospital costs before and after this policy change. Several possibilities for increased costs during overlapping surgery could include inefficient operating room utilization, longer operative times, increased complications resulting in a greater length of stay, and less efficient surgical equipment utilization. However, we hypothesized that overlapping surgery would not be associated with higher total hospital costs and that the policy change would not result in any cost reduction.

\section{Methods}

Approval was obtained from the institutional review board prior to data collection. The data collection methods for this study were previously published. ${ }^{9}$ The pre-policy change period extended from June 1, 2014, to October 31, 2014 , and the post-policy change period lasted from June 1,2016 , to October 31, 2016. The full pre- and postchange policies can be found in our prior publication, ${ }^{9}$ but the most substantive modifications were 1) a new rule mandating that an overlapping case could not be initiated until after the completion of all critical portions of the first case, and 2) another rule dictating that the attending physician could not return to the first case after beginning a second (barring unforeseen circumstances). We dichotomized all neurosurgical cases involving patients 10 years and older that were performed by any of 4 neurosurgeons at our institution who were authorized to run overlapping surgery during both timeframes into overlapping and nonoverlapping for both periods. An overlapping case was defined as any case where 2 patients under the care of a single attending surgeon were under anesthesia at the same time for any period. For this study, emergency cases were excluded from our analysis because they were almost exclusively nonoverlapping and because of the high rate of adverse events due to the critical condition of the patient. Emergency operations were defined as cases begun less than 24 hours after being added to the schedule, because of the life-threatening nature of the condition being treated.

In addition to the data previously described, total hospital cost data, excluding physician fees, were obtained from the VDO. Cost was further divided into 5 broad categories: facility fees including operating room costs, pharmacy costs, supply and implant costs, imaging costs, and laboratory costs. Because of the proprietary nature of VDO data, actual dollar values are embargoed. All financial data in the study are reported as a proportion of average total hospital cost for all admissions in the cohort. Cases in which VDO data were unavailable were excluded, as were emergency cases or cases involving surgeries performed during the weekend and those involving patients younger than 10 years. Cases in which multiple surgeries were performed during the same hospitalization were only counted once; the overlapping status for these cases was based on the operation that occurred earliest during the hospitalization, although complications during the entire hospitalization were taken into account, as were costs for all surgical procedures performed during these admissions. All 2014 cost values were adjusted for medical inflation over the study period at a rate of $6.52 \%$ based on US Bureau of Labor Statistics data (https://www.bls.gov/cpi/).

\section{Statistical Analysis}

Our study focused on analysis of cost-related data. For our univariate analysis, we utilized the Student t-test, 1-way ANOVA, or a Pearson bivariate cross-correlation analysis. Individual cost categories were analyzed with relation to both the policy change and overlap status. Total hospital cost was further analyzed in relation to all collected variables of interest. We also performed a multiple linear regression analysis of total hospital costs. Variables included in the linear regression model were those with $\mathrm{p}$ $<0.2$ on univariate analysis. The length-of-stay variable was excluded because of its direct influence on cost and its high correlation with total hospital costs in our analysis. For all analyses, $p<0.05$ was considered statistically significant. All statistical analysis was performed using IBM SPSS version 25.0 (IBM Corp).

\section{Results}

\section{Patient Cohort}

Of 695 cases performed by the 4 neurosurgeons during the study period, 625 (89.9\%) met the criteria for inclusion. Twenty-three cases were excluded because they were classified as emergency, 16 cases occurred on a weekend, 2 cases were excluded because the patient was younger than 10 years at the time of surgery, and 1 elective case was excluded because it overlapped with an emergency case. Twenty-two additional cases were excluded because they were performed during the same hospitalization as another case. VDO data for 6 cases were unavailable, and these cases were excluded as well. Three hundred sixty-two admissions occurred prior to the overlapping surgery policy change $(57.9 \%)$, and 263 occurred after the policy change $(42.1 \%)$.

\section{Univariate Analysis}

The incidence of overlapping surgery decreased significantly after the policy change (pre-policy change $46.7 \%$ vs post-policy change $27.4 \%$, $\mathrm{p}<0.001$; Table 1 ), but other variables such as case type did not change $(\mathrm{p}$ $=0.644)$. Overlapping surgery was not associated with any significant difference in any of the 5 individual cost categories (Table 2). After the policy change, laboratory costs were significantly increased $(\mathrm{p}<0.001)$, but no other cost categories were significantly affected (Table 3 ). Complications were significantly associated with higher mean hospital costs, both overall $(\mathrm{p}<0.001)$ and serious $(\mathrm{p}<$ 0.001) complications (Table 4). Patients who experienced higher-grade complications (defined as those having grade II or higher based on our previously described scale ${ }^{7}$ ) also incurred significantly higher hospital costs $(\mathrm{p}<0.001)$. Costs for hospitalizations associated with urgent surgery were higher than those associated with elective surgery $(\mathrm{p}<0.001)$.

Patients with preoperative tobacco use had significantly 
TABLE 1. Univariate analysis of policy change

\begin{tabular}{|c|c|c|c|}
\hline Variable & $\begin{array}{c}\text { Pre-Policy } \\
\text { Change } \\
(n=362)\end{array}$ & $\begin{array}{c}\text { Post-Policy } \\
\text { Change } \\
(n=263)\end{array}$ & $\mathrm{p}$ Value \\
\hline Age, yrs $\pm S D$ & $53.7 \pm 16.2$ & $49.1 \pm 17.6$ & 0.001 \\
\hline Male sex & $182(50.3)$ & $105(39.9)$ & 0.010 \\
\hline Race/ethnicity & & & 0.646 \\
\hline Caucasian & $324(89.5)$ & $226(85.9)$ & \\
\hline Hispanic/Latino & $19(5.2)$ & $17(6.5)$ & \\
\hline African American & $1(0.3)$ & $2(0.8)$ & \\
\hline Asian & $4(1.1)$ & $3(1.1)$ & \\
\hline American Indian & $3(0.8)$ & $2(0.8)$ & \\
\hline Hawaiian/Pacific Islander & $4(1.1)$ & $2(0.8)$ & \\
\hline Other/unknown & $7(1.9)$ & $11(4.2)$ & \\
\hline $\mathrm{BMI} \pm \mathrm{SD}$ & $28.6 \pm 6.8$ & $28.7 \pm 6.9$ & 0.902 \\
\hline Insurance type & & & 0.031 \\
\hline Private & $195(53.9)$ & $153(58.2)$ & \\
\hline Medicaid/self-pay & $28(7.7)$ & $32(12.2)$ & \\
\hline Medicare & $139(38.4)$ & $78(29.7)$ & \\
\hline ASA class & & & $<0.001$ \\
\hline | or II & $166(45.9)$ & $152(57.8)$ & \\
\hline III & $176(48.6)$ & $86(32.7)$ & \\
\hline IV or V & $20(5.5)$ & $25(9.5)$ & \\
\hline Tobacco use & $38(10.5)$ & $28(10.6)$ & 0.952 \\
\hline Alcohol use & $119(32.9)$ & $97(36.9)$ & 0.298 \\
\hline Illicit drug use & $21(5.8)$ & $17(6.5)$ & 0.732 \\
\hline Corticosteroid use & $55(15.2)$ & $38(14.4)$ & 0.796 \\
\hline Cardiovascular comorbidity & $56(15.5)$ & 35 (13.3) & 0.449 \\
\hline Pulmonary comorbidity & $116(32.0)$ & $88(33.5)$ & 0.709 \\
\hline Hepatobiliary comorbidity & $14(3.9)$ & $6(2.3)$ & 0.266 \\
\hline Renal comorbidity & $6(1.7)$ & $8(3.0)$ & 0.248 \\
\hline Neurological comorbidity & $60(16.6)$ & $24(9.1)$ & 0.007 \\
\hline Oncological comorbidity & $44(12.2)$ & $12(4.6)$ & 0.001 \\
\hline Diabetes & $38(10.5)$ & $31(11.8)$ & 0.611 \\
\hline Hypertension & $131(36.2)$ & $82(31.2)$ & 0.192 \\
\hline Surgery category & & & 0.644 \\
\hline $\begin{array}{l}\text { Craniotomy for tumor, } \\
\text { simple }\end{array}$ & $59(16.3)$ & $39(14.8)$ & \\
\hline $\begin{array}{l}\text { Craniotomy for tumor, } \\
\text { complex }\end{array}$ & $17(4.7)$ & $18(6.8)$ & \\
\hline $\begin{array}{l}\text { Craniotomy for aneurysm/ } \\
\text { vascular malformation }\end{array}$ & $19(5.2)$ & $7(2.7)$ & \\
\hline Craniotomy for other & $18(5.0)$ & $17(6.5)$ & \\
\hline Burr hole craniotomy & $12(3.3)$ & $6(2.3)$ & \\
\hline Noninstrumented spine & $44(12.2)$ & $36(13.7)$ & \\
\hline Instrumented spine & $71(19.6)$ & $59(22.4)$ & \\
\hline $\begin{array}{l}\text { Transnasal approach for } \\
\text { tumor }\end{array}$ & $31(8.6)$ & $20(7.6)$ & \\
\hline Shunt & $42(11.6)$ & $32(12.2)$ & \\
\hline Miscellaneous & $49(13.5)$ & $29(11.0)$ & \\
\hline Senior attending* & $180(49.7)$ & $133(50.6)$ & 0.834 \\
\hline Overlapping surgery & $169(46.7)$ & $72(27.4)$ & $<0.001$ \\
\hline
\end{tabular}

» CONTINUED FROM PREVIOUS COLUMN

TABLE 1. Univariate analysis of policy change

\begin{tabular}{|c|c|c|c|}
\hline Variable & $\begin{array}{l}\text { Pre-Policy } \\
\text { Change } \\
(n=362)\end{array}$ & $\begin{array}{c}\text { Post-Policy } \\
\text { Change } \\
(n=263)\end{array}$ & $p$ Value \\
\hline Scheduling type & & & 0.005 \\
\hline Elective & $319(88.1)$ & $249(94.7)$ & \\
\hline Urgent & $43(11.9)$ & $14(5.3)$ & \\
\hline Assistant training level & & & $<0.001$ \\
\hline PGY-1 & $5(1.4)$ & $5(1.9)$ & \\
\hline PGY-2 & $26(7.2)$ & $6(2.3)$ & \\
\hline PGY-3 & $4(1.1)$ & $15(5.7)$ & \\
\hline PGY-4 & $6(1.7)$ & $11(4.2)$ & \\
\hline PGY-5 & $88(24.3)$ & $34(12.9)$ & \\
\hline PGY-6 & $74(20.4)$ & $22(8.4)$ & \\
\hline PGY-7 & $114(31.5)$ & $80(30.4)$ & \\
\hline Fellow & $45(12.4)$ & $90(34.2)$ & \\
\hline Op length, mins \pm SD & $277.3 \pm 137.7$ & $289.6 \pm 128.8$ & 0.261 \\
\hline Overlap time, mins \pm SD & $194.2 \pm 102.5$ & $156.8 \pm 111.2$ & 0.012 \\
\hline $\mathrm{EBL}, \mathrm{ml} \pm \mathrm{SD}$ & $199.1 \pm 398.2$ & $197.4 \pm 280.0$ & 0.952 \\
\hline LOS, days \pm SD & $4.3 \pm 4.8$ & $4.1 \pm 3.9$ & 0.654 \\
\hline Discharge destination & & & 0.248 \\
\hline Home & $251(69.3)$ & $196(74.5)$ & \\
\hline Home health & $16(4.4)$ & $12(4.6)$ & \\
\hline Acute rehab & $54(14.9)$ & $39(14.8)$ & \\
\hline SNF & $28(7.7)$ & $8(3.0)$ & \\
\hline LTAC & $8(2.2)$ & $6(2.3)$ & \\
\hline Death & $3(0.8)$ & $2(0.8)$ & \\
\hline Other & $2(0.6)$ & $0(0)$ & \\
\hline Intraop complications & $21(5.8)$ & $14(5.3)$ & 0.798 \\
\hline Intraop complication grade & & & 0.814 \\
\hline 0 & 341 (94.2) & $249(94.7)$ & \\
\hline I & $17(4.7)$ & $13(4.9)$ & \\
\hline II & $1(0.3)$ & $0(0)$ & \\
\hline III & $2(0.6)$ & $1(0.4)$ & \\
\hline IV & $1(0.3)$ & $0(0)$ & \\
\hline Postop complications & $67(18.5)$ & $51(19.4)$ & 0.781 \\
\hline Postop complication grade & & & 0.734 \\
\hline 0 & $295(81.5)$ & $212(80.6)$ & \\
\hline I & $36(9.9)$ & $29(11.0)$ & \\
\hline II & $5(1.4)$ & $7(2.7)$ & \\
\hline III & $24(6.6)$ & $14(5.3)$ & \\
\hline IV & $2(0.6)$ & $1(0.4)$ & \\
\hline 30-day complications & $29(8.0)$ & $18(6.8)$ & 0.585 \\
\hline 30-day complication grade & & & 0.368 \\
\hline 0 & $333(92.0)$ & $245(93.2)$ & \\
\hline I & $12(3.3)$ & $3(1.1)$ & \\
\hline II & $8(2.2)$ & $7(2.7)$ & \\
\hline III & $8(2.2)$ & $8(3.0)$ & \\
\hline IV & $1(0.3)$ & $0(0)$ & \\
\hline Any complication & $97(26.8)$ & $72(27.4)$ & 0.872 \\
\hline Serious complication $†$ & $48(13.3)$ & $37(14.1)$ & 0.771 \\
\hline
\end{tabular}

CONTINUED ON PAGE $906 »$ 
» CONTINUED FROM PAGE 905

TABLE 1. Univariate analysis of policy change

\begin{tabular}{cccc}
\hline Variable & $\begin{array}{c}\text { Pre-Policy } \\
\text { Change } \\
(\mathrm{n}=362)\end{array}$ & $\begin{array}{c}\text { Post-Policy } \\
\text { Change } \\
(\mathrm{n}=263)\end{array}$ & p Value \\
\cline { 1 - 3 } Worst complication grade & & \multicolumn{2}{c}{0.862} \\
\cline { 1 - 3 } I & $265(73.2)$ & $191(72.6)$ & \\
\cline { 1 - 3 } I & $49(13.5)$ & $35(13.3)$ & \\
\cline { 1 - 3 } III & $16(4.4)$ & $13(4.9)$ & \\
IV & $28(7.7)$ & $23(8.7)$ & \\
\cline { 1 - 2 } & $4(1.1)$ & $1(0.4)$ &
\end{tabular}

$\mathrm{EBL}=$ estimated blood loss; $\mathrm{LOS}=$ length of stay; $\mathrm{LTAC}=$ long-term acute care facility; PGY = postgraduate year; rehab = rehabilitation; SNF = skilled nursing facility.

Values are presented as the number of cases (\%) unless stated otherwise. Boldface type indicates statistical significance.

${ }^{*} \geq 20$ years postresidency.

$\dagger$ Complications of grade II or higher.

higher hospital costs than those who did not $(p=0.024)$, although pulmonary comorbidities actually appeared to be associated with lower costs $(\mathrm{p}=0.036)$. Patients in higher American Society of Anesthesiologists (ASA) classes incurred significantly higher hospital costs $(\mathrm{p}<0.001)$, with ASA class $V$ patients having a mean cost nearly 5 times higher than those in ASA class I. Surgery type was significantly associated with hospital cost $(\mathrm{p}<0.001)$, with craniotomy for aneurysm/vascular malformation having the highest mean cost and noninstrumented spine surgery having the lowest mean cost. Discharge destination was also significantly associated with cost $(\mathrm{p}<0.001)$, with home discharge having the lowest mean hospital costs and death having the highest. In our Pearson bivariate crosscorrelation analysis, hospital cost was significantly correlated with longer operating room time (correlation coefficient $0.306, \mathrm{p}<0.001$ ), higher intraoperative blood loss (correlation coefficient $0.263, \mathrm{p}<0.001$ ), and length of stay (correlation coefficient 0.718, $\mathrm{p}<0.001$; Table 5).

\section{Multivariate Analysis}

In our multivariate linear regression model, the overlapping surgery policy change was not associated with any change in mean hospital costs in our cohort (Table 6; B $=0.041,95 \% \mathrm{CI}-0.106$ to 0.189 ). The occurrence of any

TABLE 2. Univariate cost analysis of overlapping surgery

\begin{tabular}{lccc}
\hline & \multicolumn{2}{c}{ Mean Cost \pm SD } & \\
\cline { 2 - 3 } Cost Category & $\begin{array}{c}\text { Nonoverlapping } \\
(\mathrm{n}=384)\end{array}$ & $\begin{array}{c}\text { Overlapping } \\
(\mathrm{n}=241)\end{array}$ & $\begin{array}{c}\mathrm{p} \\
\text { Value }\end{array}$ \\
\hline Facility & $0.559 \pm 0.701$ & $0.496 \pm 0.552$ & 0.239 \\
\hline Pharmacy & $0.122 \pm 0.185$ & $0.105 \pm 0.136$ & 0.226 \\
\hline Supply/implant & $0.259 \pm 0.369$ & $0.253 \pm 0.353$ & 0.829 \\
\hline Imaging & $0.026 \pm 0.061$ & $0.023 \pm 0.072$ & 0.637 \\
\hline Lab & $0.073 \pm 0.134$ & $0.061 \pm 0.168$ & 0.302 \\
\hline
\end{tabular}

TABLE 3. Univariate cost analysis of policy implementation

\begin{tabular}{lccr}
\hline & \multicolumn{2}{c}{ Mean Cost \pm SD } & \\
\cline { 2 - 3 } Cost Category & $\begin{array}{c}\text { Pre-Policy } \\
\text { Change }(n=362)\end{array}$ & $\begin{array}{c}\text { Post-Policy } \\
\text { Change }(n=263)\end{array}$ & $\begin{array}{c}p \\
\text { Value }\end{array}$ \\
\hline Facility & $0.542 \pm 0.723$ & $0.524 \pm 0.527$ & 0.737 \\
\hline Pharmacy & $0.112 \pm 0.181$ & $0.120 \pm 0.147$ & 0.577 \\
\hline Supply/implant & $0.267 \pm 0.378$ & $0.242 \pm 0.341$ & 0.396 \\
\hline Imaging & $0.026 \pm 0.082$ & $0.023 \pm 0.032$ & 0.522 \\
\hline Lab & $0.046 \pm 0.148$ & $0.099 \pm 0.142$ & $<0.001$ \\
\hline
\end{tabular}

Boldface type indicates statistical significance.

complication during the hospitalization was associated with a significantly higher total hospital cost $(B=0.321$, $95 \%$ CI 0.142 to 0.500 ), as was a surgery that was categorized as urgent during the hospitalization $(\mathrm{B}=0.385,95 \%$ CI 0.136 to 0.635 ). Tobacco use remained significantly associated with higher costs in our regression model $(\mathrm{B}=$ $0.232,95 \%$ CI 0.011 to 0.453 ), whereas preoperative steroid use was associated with lower cost $(\mathrm{B}=-0.239,95 \%$ CI -0.437 to -0.040 ). Compared with the reference of instrumented spine surgery, craniotomy for simple tumor ( $\mathrm{B}=-0.463,95 \% \mathrm{CI}-0.698$ to -0.228 ), craniotomy for complex tumor $(\mathrm{B}=-0.447,95 \% \mathrm{CI}-0.799$ to -0.095$)$, craniotomy for other $(\mathrm{B}=-0.493,95 \% \mathrm{CI}-0.822$ to $-0.164)$, and noninstrumented spine surgery $(\mathrm{B}=-0.514$, $95 \%$ CI -0.770 to -0.258 ) were all associated with lower hospital costs. Compared with discharge home, all other discharge destinations apart from "other" were associated with higher costs (all $\mathrm{p}<0.05$ ). Finally, compared with a reference ASA class of II, ASA classes of III $(B=0.172$, 95\% CI $0.015-0.330)$ and IV (B $=0.626,95 \%$ CI 0.314 to 0.939 ) were associated with higher costs.

\section{Discussion}

A number of studies have examined the association of overlapping surgery with adverse events, $7,13,18,20$ and although the vast majority of these have found no association between the practice and increased complication rate, a number of centers, including our own, have adjusted their policies in response to the increasing controversy. Our study represents the first analysis of costs related to overlapping surgery in a broad neurosurgical case mix and the first examining the influence of a more restrictive policy on costs.

Our results suggest that a more stringent overlapping surgery policy did not lead to cost savings in our cohort, and overlapping surgery was not associated with higher or lower costs in and of itself. The variables that did appear to lead to higher hospitalization costs were largely unsurprising - complications, ${ }^{5,12}$ emergency surgery, ${ }^{11}$ operating room time ${ }^{15}$ surgery type,${ }^{6}$ inability to discharge home,${ }^{17}$ higher ASA class,${ }^{19}$ and tobacco use ${ }^{10,16}$ - and have all previously been shown to be significantly associated with hospital costs. Our finding that preoperative steroid use was associated with lower costs was somewhat surprising, although some evidence suggests that such use may reduce length of stay in patients undergoing crani- 
TABLE 4. Analysis of total hospital costs

\begin{tabular}{|c|c|c|}
\hline Variable (no. of cases) & Mean Cost \pm SD & p Value \\
\hline Policy status & & 0.873 \\
\hline Before change (362) & $0.994 \pm 1.237$ & \\
\hline After change (263) & $1.009 \pm 0.994$ & \\
\hline Complication & & $<0.001$ \\
\hline No (456) & $0.735 \pm 0.741$ & \\
\hline Yes (169) & $1.716 \pm 1.623$ & \\
\hline Serious complication (grade $\geq I I$ ) & & $<0.001$ \\
\hline No $(540)$ & $0.853 \pm 0.870$ & \\
\hline Yes (85) & $1.937 \pm 1.942$ & \\
\hline Maximum complication grade & & $<0.001$ \\
\hline None (456) & $0.735 \pm 0.741$ & \\
\hline I (84) & $1.493 \pm 1.189$ & \\
\hline II (29) & $1.451 \pm 2.235$ & \\
\hline III (51) & $2.053 \pm 1.676$ & \\
\hline IV (5) & $3.563 \pm 1.980$ & \\
\hline 30-day readmission & & 0.243 \\
\hline No (578) & $0.985 \pm 1.139$ & \\
\hline Yes (47) & $1.187 \pm 1.144$ & \\
\hline Intraop complication & & $<0.001$ \\
\hline No $(590)$ & $0.925 \pm 1.052$ & \\
\hline Yes (35) & $2.262 \pm 1.719$ & \\
\hline Postop complication & & $<0.001$ \\
\hline No (507) & $0.766 \pm 0.765$ & \\
\hline Yes (118) & $2.007 \pm 1.772$ & \\
\hline Attending PGYs in practice & & 0.534 \\
\hline$<20(312)$ & $0.972 \pm 1.033$ & \\
\hline$\geq 20(313)$ & $1.028 \pm 1.239$ & \\
\hline Surgery urgency & & $<0.001$ \\
\hline Elective (568) & $0.943 \pm 1.011$ & \\
\hline Urgent (57) & $1.570 \pm 1.940$ & \\
\hline \multicolumn{3}{|l|}{ Assistant level of training } \\
\hline PGY 1-6 (296) & $0.932 \pm 1.108$ & 0.157 \\
\hline PGY 7 or fellow (329) & $1.061 \pm 1.166$ & \\
\hline Patient sex & & 0.830 \\
\hline Male (287) & $1.011 \pm 1.159$ & \\
\hline Female (338) & $0.991 \pm 1.126$ & \\
\hline Overlap status & & 0.282 \\
\hline Nonoverlapping (384) & $1.039 \pm 1.215$ & \\
\hline Overlapping (241) & $0.938 \pm 1.009$ & \\
\hline Tobacco use & & 0.024 \\
\hline No (559) & $0.965 \pm 1.049$ & \\
\hline Yes (66) & $1.300 \pm 1.712$ & \\
\hline Alcohol use & & 0.368 \\
\hline No (409) & $0.970 \pm 1.118$ & \\
\hline Yes (216) & $1.057 \pm 1.182$ & \\
\hline Illicit drug use & & 0.705 \\
\hline No (587) & $0.996 \pm 1.128$ & \\
\hline Yes (38) & $1.068 \pm 1.335$ & \\
\hline
\end{tabular}

» CONTINUED FROM PREVIOUS COLUMN

TABLE 4. Analysis of total hospital costs

\begin{tabular}{|c|c|c|}
\hline Variable (no. of cases) & Mean Cost \pm SD & $p$ Value \\
\hline Preop corticosteroid use & & 0.078 \\
\hline No (532) & $1.034 \pm 1.206$ & \\
\hline Yes (93) & $0.808 \pm 0.618$ & \\
\hline Cardiovascular comorbidity & & 0.380 \\
\hline No (534) & $0.984 \pm 1.128$ & \\
\hline Yes (91) & $1.097 \pm 1.208$ & \\
\hline Pulmonary comorbidity & & 0.036 \\
\hline No (421) & $1.067 \pm 1.227$ & \\
\hline Yes (204) & $0.863 \pm 0.924$ & \\
\hline Neurological comorbidity & & 0.383 \\
\hline No (541) & $0.984 \pm 1.123$ & \\
\hline Yes (84) & $1.101 \pm 1.249$ & \\
\hline Hepatobiliary comorbidity & & 0.874 \\
\hline No $(605)$ & $0.999 \pm 1.147$ & \\
\hline Yes (20) & $1.040 \pm 0.928$ & \\
\hline Renal comorbidity & & 0.872 \\
\hline No (611) & $1.001 \pm 1.146$ & \\
\hline Yes (14) & $0.951 \pm 0.867$ & \\
\hline Oncological comorbidity & & 0.288 \\
\hline No (569) & $0.985 \pm 1.104$ & \\
\hline Yes (56) & $1.155 \pm 1.460$ & \\
\hline Diabetes & & 0.156 \\
\hline No (556) & $0.977 \pm 1.108$ & \\
\hline Yes (69) & $1.184 \pm 1.364$ & \\
\hline Hypertension & & 0.079 \\
\hline No (412) & $0.942 \pm 1.035$ & \\
\hline Yes (213) & $1.111 \pm 1.315$ & \\
\hline Surgery day of week & & 0.642 \\
\hline Monday (183) & $0.903 \pm 1.105$ & \\
\hline Tuesday (68) & $0.997 \pm 1.249$ & \\
\hline Wednesday (48) & $1.094 \pm 1.071$ & \\
\hline Thursday (182) & $1.003 \pm 1.131$ & \\
\hline Friday (144) & $1.089 \pm 1.269$ & \\
\hline Surgery type & & $<0.001$ \\
\hline Craniotomy for tumor, simple (98) & $0.977 \pm 0.977$ & \\
\hline Craniotomy for tumor, complex (35) & $1.356 \pm 1.378$ & \\
\hline $\begin{array}{l}\text { Craniotomy for aneurysm/vascular } \\
\text { malformation (26) }\end{array}$ & $2.146 \pm 2.476$ & \\
\hline Craniotomy for other (35) & $0.810 \pm 0.715$ & \\
\hline Burr hole craniotomy (18) & $1.173 \pm 1.281$ & \\
\hline Noninstrumented spine (80) & $0.323 \pm 0.207$ & \\
\hline Instrumented spine (130) & $1.373 \pm 1.126$ & \\
\hline Transnasal approach for tumor (51) & $0.573 \pm 0.367$ & \\
\hline Shunt procedure (74) & $0.783 \pm 0.955$ & \\
\hline Miscellaneous (78) & $1.092 \pm 1.176$ & \\
\hline
\end{tabular}

CONTINUED ON PAGE $908 »$ 
» CONTINUED FROM PAGE 907

TABLE 4. Analysis of total hospital costs

\begin{tabular}{|c|c|c|}
\hline Variable (no. of cases) & Mean Cost \pm SD & p Value \\
\hline Attending & & 0.627 \\
\hline $1(203)$ & $0.974 \pm 1.094$ & \\
\hline $2(102)$ & $0.955 \pm 1.048$ & \\
\hline $3(110)$ & $1.129 \pm 1.469$ & \\
\hline $4(210)$ & $0.979 \pm 1.028$ & \\
\hline Discharge destination & & $<0.001$ \\
\hline Home (447) & $0.661 \pm 0.602$ & \\
\hline Home health (28) & $1.260 \pm 1.154$ & \\
\hline Rehab (93) & $1.798 \pm 1.449$ & \\
\hline SNF (36) & $1.555 \pm 1.742$ & \\
\hline LTAC (14) & $3.523 \pm 1.958$ & \\
\hline Death (5) & $3.898 \pm 1.546$ & \\
\hline Other (2) & $1.134 \pm 0.662$ & \\
\hline Insurance type & & 0.671 \\
\hline Private (348) & $0.982 \pm 1.047$ & \\
\hline Medicaid/self-pay (60) & $1.124 \pm 1.761$ & \\
\hline Medicare (217) & $0.995 \pm 1.069$ & \\
\hline Race/ethnicity & & 0.212 \\
\hline Caucasian (550) & $0.971 \pm 1.132$ & \\
\hline Hispanic/Latino (36) & $1.054 \pm 1.007$ & \\
\hline African American (3) & $1.597 \pm 1.819$ & \\
\hline Asian (7) & $0.743 \pm 0.234$ & \\
\hline American Indian (5) & $1.794 \pm 1.107$ & \\
\hline Hawaiian/Pacific Islander (6) & $1.824 \pm 2.704$ & \\
\hline Other/unknown (18) & $1.293 \pm 0.860$ & \\
\hline ASA classification & & $<0.001$ \\
\hline I (31) & $0.635 \pm 0.690$ & \\
\hline II (287) & $0.720 \pm 0.640$ & \\
\hline III (262) & $1.126 \pm 1.177$ & \\
\hline IV (42) & $2.240 \pm 2.174$ & \\
\hline $\mathrm{V}(3)$ & $3.186 \pm 2.694$ & \\
\hline
\end{tabular}

ANOVA and the Student t-test were used for comparisons. Boldface type indicates statistical significance.

otomy for tumor, which may partially explain this association. $^{2}$ The finding that laboratory costs for our cohort were significantly higher after the policy change was also unexpected, although we believe that this is more likely related to the introduction of new technologies to our hospital system (e.g., rotational thromboelastometry). ${ }^{8}$ Case mix prior to the policy change and after the policy change was not significantly different in our cohort, as previously reported. ${ }^{9}$ The total number of cases performed before the policy change (378) was more than that performed after the change (275), and thus the sum total of direct hospital costs after the policy change was only $73.4 \%$ of costs before the policy change. The more restrictive policy did appear effective in limiting overlapping surgery, as the rate of overlapping cases declined from $46 \%$ prechange to $26.9 \%$ postchange. ${ }^{9}$

Our study has several limitations. As with any retrospective study, ours is vulnerable to confounding. Our study was limited to a single center and may not be generalizable to other practices. The inability to release dollar values makes our data relatively harder to interpret, although the uniform transformation of our values throughout the study should ensure that our findings were not significantly affected. Finally, our study only examines direct costs, and we cannot therefore make any conclusions regarding the effect of this policy change on indirect costs associated with care.

Despite these limitations, we believe that our data accurately indicate that the policy changes aimed at restricting overlapping surgery at our center have not resulted in any cost savings. In fact, our results implicate complications as a major cost driver for neurosurgical hospitalizations, and the fact that overlapping surgery was not associated with higher costs further supports the conclusion from other studies that overlapping surgery does not result in higher complication rates. Furthermore, less-efficient resource utilization that has been predicted to occur with overlapping surgery, which could result in longer operating room times or lengths of stay, was not observed in this analysis.

\section{Conclusions}

In our cohort, a more restrictive overlapping surgery policy was not associated with any significant reductions in hospital costs. Additional investigation is needed to further characterize whether limiting overlapping surgery has any economic healthcare benefits.

\section{Acknowledgments}

We thank Kristin Kraus, MSc, for editorial assistance with this

TABLE 5. Pearson bivariate cross-correlation of continuous variables with total hospital costs

\begin{tabular}{lcccccc}
\hline \multicolumn{1}{c}{ Variable } & Total Hospital Costs & OR Time & Patient Age & BMl & Intraop EBL & LOS \\
\hline Total hospital costs & 1 & $0.306^{*}$ & -0.008 & -0.014 & $0.263^{*}$ & $0.718^{*}$ \\
\hline OR time & & 1 & 0.005 & -0.006 & $0.552^{*}$ & $0.385^{*}$ \\
\hline Patient age & & 1 & -0.032 & 0.010 & 0.019 \\
\hline BMI & & & 1 & 0.015 & -0.035 \\
\hline Intraop EBL & & & & 1 & $0.257^{*}$ \\
\hline LOS & & & & & 1 \\
\hline
\end{tabular}


TABLE 6. Multivariate linear regression of factors affecting total hospital cost

\begin{tabular}{|c|c|c|c|}
\hline Variable & $\mathrm{B}(\mathrm{SE})$ & $95 \% \mathrm{Cl}$ & $\begin{array}{c}\mathrm{p} \\
\text { Value }\end{array}$ \\
\hline Post-policy change & $0.041(0.075)$ & -0.106 to 0.189 & 0.582 \\
\hline Any complication & $0.321(0.091)$ & 0.142 to 0.500 & $<0.001$ \\
\hline Urgent surgery & $0.385(0.127)$ & 0.136 to 0.635 & 0.002 \\
\hline $\begin{array}{l}\text { Chief resident/fellow } \\
\text { assistant }\end{array}$ & $0.002(0.076)$ & -0.148 to 0.151 & 0.981 \\
\hline Overlapping surgery & $-0.080(0.073)$ & -0.223 to 0.063 & 0.273 \\
\hline Tobacco use & $0.232(0.112)$ & 0.011 to 0.453 & 0.040 \\
\hline Preop steroid use & $-0.239(0.101)$ & -0.437 to -0.040 & 0.019 \\
\hline Pulmonary comorbidity & $-0.140(0.077)$ & -0.290 to 0.011 & 0.068 \\
\hline Diabetes & $-0.027(0.118)$ & -0.258 to 0.205 & 0.820 \\
\hline Hypertension & $0.033(0.079)$ & -0.121 to 0.188 & 0.673 \\
\hline OR time, mins & $0.002(0.000)$ & 0.001 to 0.003 & $<0.001$ \\
\hline Intraop EBL (ml) & $0.000(0.000)$ & 0.000 to 0.000 & 0.067 \\
\hline \multicolumn{4}{|l|}{ Surgery type } \\
\hline Instrumented spine & Ref & Ref & \\
\hline $\begin{array}{l}\text { Craniotomy for } \\
\text { tumor, simple }\end{array}$ & $-0.463(0.120)$ & -0.698 to -0.228 & $<0.001$ \\
\hline $\begin{array}{l}\text { Craniotomy for } \\
\text { tumor, complex }\end{array}$ & $-0.447(0.179)$ & -0.799 to -0.095 & 0.013 \\
\hline $\begin{array}{l}\text { Craniotomy for an- } \\
\text { eurysm/vascular } \\
\text { malformation }\end{array}$ & $0.226(0.197)$ & -0.162 to -0.614 & 0.252 \\
\hline Craniotomy for other & $-0.493(0.167)$ & -0.822 to -0.164 & 0.003 \\
\hline Burr hole craniotomy & $-0.386(0.233)$ & -0.844 to 0.071 & 0.098 \\
\hline $\begin{array}{l}\text { Noninstrumented } \\
\text { spine }\end{array}$ & $-0.514(0.130)$ & -0.770 to -0.258 & $<0.001$ \\
\hline $\begin{array}{l}\text { Transnasal ap- } \\
\text { proach for tumor }\end{array}$ & $-0.293(0.156)$ & -0.600 to 0.013 & 0.060 \\
\hline Shunt procedure & $-0.220(0.141)$ & -0.496 to 0.056 & 0.118 \\
\hline Miscellaneous & $-0.117(0.143)$ & -0.398 to 0.164 & 0.413 \\
\hline \multicolumn{4}{|l|}{ Discharge destination } \\
\hline Home & Ref & Ref & \\
\hline Home health & $0.380(0.171)$ & 0.044 to 0.716 & 0.027 \\
\hline Rehab & $0.643(0.113)$ & 0.421 to 0.866 & $<0.001$ \\
\hline SNF & $0.500(0.157)$ & 0.192 to 0.809 & 0.002 \\
\hline LTAC & $2.350(0.256)$ & 1.849 to 2.852 & $<0.001$ \\
\hline Death & $1.758(0.429)$ & 0.916 to 2.600 & $<0.001$ \\
\hline Other & $-0.107(0.611)$ & -1.308 to 1.093 & 0.861 \\
\hline \multicolumn{4}{|l|}{ ASA classification } \\
\hline I & $0.042(0.165)$ & -0.282 to 0.366 & 0.800 \\
\hline II & Ref & Ref & \\
\hline III & $0.172(0.080)$ & 0.015 to 0.330 & 0.031 \\
\hline IV & $0.626(0.159)$ & 0.314 to 0.939 & $<0.001$ \\
\hline V & $0.735(0.534)$ & -0.314 to 1.784 & 0.169 \\
\hline
\end{tabular}

$\mathrm{SE}=$ standard error

Boldface type indicates statistical significance. paper. We also thank Jacob Kresser, BS, for his assistance with data acquisition from the VDO.

\section{References}

1. Abelson J, Saltzman J, Kowalczyk L, Allen S: Clash in the name of care. Boston Globe. October 25, 2015. (https://apps. bostonglobe.com/spotlight/clash-in-the-name-of-care/story) [Accessed on June 24, 2018]

2. Alan N, Seicean A, Seicean S, Neuhauser D, Benzel EC, Weil RJ: Preoperative steroid use and the incidence of perioperative complications in patients undergoing craniotomy for definitive resection of a malignant brain tumor. J Clin Neurosci 22:1413-1419, 2015

3. American Association of Neurological Surgeons: Position statement on intraoperative responsibility of the primary neurosurgeon. AANS.org. (http://www.aans.org/pdf/Legislative/ Neurosurgery $\% 20$ Position $\% 20$ Statement $\% 20$ on $\% 20$ Overlapping\%20Surgery\%20FINAL.pdf) [Accessed on June 25, 2018]

4. American College of Surgeons: Statement on principles. FACS.org. (https://www.facs.org/about-acs/statements/ stonprin\#anchor172771) [Accessed on June 24, 2018]

5. Dimick JB, Chen SL, Taheri PA, Henderson WG, Khuri SF, Campbell DA Jr: Hospital costs associated with surgical complications: a report from the private-sector National Surgical Quality Improvement Program. J Am Coll Surg 199:531-537, 2004

6. Gani F, Hundt J, Daniel M, Efron JE, Makary MA, Pawlik TM: Variations in hospitals costs for surgical procedures: inefficient care or sick patients? Am J Surg 213:1-9, 2017

7. Guan J, Brock AA, Karsy M, Couldwell WT, Schmidt MH, Kestle JRW, et al: Managing overlapping surgery: an analysis of 1018 neurosurgical and spine cases. J Neurosurg 127:1096-1104, 2017

8. Guan J, Cole CD, Schmidt MH, Dailey AT: Utility of intraoperative rotational thromboelastometry in thoracolumbar deformity surgery. J Neurosurg Spine 27:528-533, 2017

9. Guan J, Karsy M, Brock A, Couldwell W, Kestle J, Jensen R, et al: Impact of a more restrictive overlapping surgery policy: an analysis of pre- and postimplementation complication rates, resident involvement, and surgical wait times at a highvolume neurosurgical department. J Neurosurg [epub ahead of print November 3, 2017; DOI: 10.3171/2017.5.JNS17183]

10. Guan J, Karsy M, Schmidt M, Dailey AT, Bisson E: Multivariable analysis of factors affecting length of stay and hospital charges after atlantoaxial fusion. Cureus 9:e1173, 2017

11. Haider AH, Obirieze A, Velopulos CG, Richard P, Latif A, Scott VK, et al: Incremental cost of emergency versus elective surgery. Ann Surg 262:260-266, 2015

12. Healy MA, Mullard AJ, Campbell DA Jr, Dimick JB: Hospital and payer costs associated with surgical complications. JAMA Surg 151:823-830, 2016

13. Hyder JA, Hanson KT, Storlie CB, Glasgow A, Madde NR, Brown MJ, et al: Safety of overlapping surgery at a highvolume referral center. Ann Surg 265:639-644, 2017

14. Lee VS, Kawamoto K, Hess R, Park C, Young J, Hunter C, et al: Implementation of a value-driven outcomes program to identify high variability in clinical costs and outcomes and association with reduced cost and improved quality. JAMA 316:1061-1072, 2016

15. Mercier G, Naro G: Costing hospital surgery services: the method matters. PLoS One 9:e97290, 2014

16. Murphy CC, Incalcaterra JR, Albright HW, Correa AM, Swisher SG, Hofstetter WL: Pretreatment patient comorbidity and tobacco use increase cost and risk of postoperative complications after esophagectomy at a high-volume cancer center. J Oncol Pract 9:233-239, 2013

17. Pitzul KB, Wodchis WP, Kreder HJ, Carter MW, Jaglal SB: 
Discharge destination following hip fracture: comparative effectiveness and cost analyses. Arch Osteoporos 12:87, 2017

18. Ponce BA, Wills BW, Hudson PW, Huntley SR, Starnes AC, Watson SL, et al: Outcomes with overlapping surgery at a large academic medical center. Ann Surg [epub ahead of print], 2018

19. Puffer RC, Planchard R, Mallory GW, Clarke MJ: Patientspecific factors affecting hospital costs in lumbar spine surgery. J Neurosurg Spine 24:1-6, 2016

20. Ravi B, Pincus D, Wasserstein D, Govindarajan A, Huang A, Austin PC, et al: association of overlapping surgery with increased risk for complications following hip surgery: a population-based, matched cohort study. JAMA Intern Med 178:75-83, 2018

21. U.S. Senate Committee on Finance: Concurrent and Overlapping Surgeries: Additional Measures Warranted. Washington, DC: U.S. Senate, 2016. (http://www.finance. senate.gov/imo/media/doc/Concurrent $\% 20$ Surgeries $\% 20$ Report\%20Final.pdf) [Accessed on June 25, 2018]

22. Zygourakis CC, Sizdahkhani S, Keefe M, Lee J, Chou D, Mummaneni PV, et al: Comparison of patient outcomes and cost of overlapping versus nonoverlapping spine surgery. World Neurosurg 100:658.e8-664.e8, 2017

\section{Disclosures}

Dr. Jensen: consultant for Medtronic; and advisory boards for Pharmaco-Kinesis and Varian. Dr. Dailey: consultant for Medtronic, K2M, and Zimmer-Biomet; speaking fees from AONA; and support of non-study-related clinical or research effort from K2M. Dr. Bisson: consultant for nView.

\section{Author Contributions}

Conception and design: Schmidt, Guan, Karsy. Acquisition of data: Guan. Analysis and interpretation of data: Guan. Drafting the article: Guan, Karsy. Critically revising the article: Schmidt, Guan, Karsy, Brock, Couldwell, Kestle, Jensen, Bisson. Reviewed submitted version of manuscript: all authors. Approved the final version of the manuscript on behalf of all authors: Schmidt. Statistical analysis: Dailey.

\section{Correspondence}

Richard H. Schmidt: University of Utah, Salt Lake City, UT. neuropub@hsc.utah.edu. 